\title{
Preface
}

\section{Back to the future}

In the 50 years since Barnard's breakthrough procedure, performed in December 1967, heart transplantation has established an unrivalled position in the treatment of end-stage heart failure. No other solution can give us sustained and effective function in the long term. At a meeting to celebrate that $50^{\text {th }}$ anniversary in our own institution, we met with patients celebrating 10, 20 and even 30 years of survival, all of them with a good quality of life. But we continue to learn, evolve and improve the technique whilst facing new challenges. In particular, the role of mechanical assist devices is constantly changing. Principally, they allow us to keep alive a cohort who would have previously died. But this brings with it new challenges, both in decision-making and in surgical approach. In this issue, we bring you authorities from around the world. There is a well-researched meta-analysis comparing heart transplantation and left ventricular assist devices (LVADs). Dr. Kiran Khush provides a masterful overview of donor selection in the modern era, while Dr. Andre Simon tells us of current approaches to both donor organ retrieval and preservation. Johan Van Cleemput shows us how to maintain our patients and obtain the very best results over many years.

The particular problem of ventricular assist device explantation is beautifully illustrated for us by Dr. Stefan Schuler and his colleagues. With our background, we have placed particular emphasis on transplantation in children and adults with congenital heart disease. Dr. Leonard Bailey starts us off with his unique perspective on neonatal and infant transplants, whilst Dr. Anne Dipchand gives us an overview of the current state of transplantation across all children. Dr. David Morales repeats that same perspective for adults with congenital heart disease. Then Dr. Roland Hetzer from Berlin provides us the benefit of his huge experience in paediatric bridge to transplantation.

As we look to the future, Dr. Jon Kobashigawa gives us a wonderful insight into where we are heading across the entire field of cardiac transplantation. Finally, Dr. Stephen Large and his colleagues introduce us to the hugely exciting field of donation after circulatory death (DCD) heart to retrieval and transplantation. We are grateful to Editor-in-Chief Tristan Yan for inviting us to serve as guest editors for this special issue on cardiac transplantation.

\section{Acknowledgements}

None.

John H. Dark

Institute of Cellular Medicine, Faculty of Medical Sciences, Newcastle University, Newcastle upon Tyne, UK.

(Email: j.b.dark@ncl.ac.uk)

Asif Hasan

Department of Paediatric Cardiac Surgery, Cardiopulmonary Transplant Unit, Freeman Hospital, Newcastle upon Tyne, UK.

(Email:asif.basan@nuth.nbs.uk)

doi: $10.21037 /$ acs.2018.01.02

Conflicts of Interest: The authors have no conflicts of interest to declare.

View this article at: http://dx.doi.org/10.21037/acs.2018.01.02 